\title{
EVALUATION OF THE VIIRS LAND ALGORITHMS AT LAND PEATE
}

\author{
Robert E. Wolfe ${ }^{1}$, Sadashiva Devadiga ${ }^{2}$, Gang Y ${ }^{2}$, Edward J. Masuoka ${ }^{1}$, Robert J. Schweiss ${ }^{1}$ \\ 1. NASA Goddard Space Flight Center, Greenbelt MD. \\ 2. Sigma Space Corporation, NASA Goddard Space Flight Center, Greenbelt MD
}

\begin{abstract}
The Land Product Evaluation and Algorithm Testing Element (Land PEATE), a component of the Science Data Segment of the National Polar-orbiting Operational Environmental Satellite System (NPOESS) Preparatory Project (NPP), is being developed at the NASA Goddard Space Flight Center (GSFC). The primary task of the Land PEATE is to assess the quality of the Visible Infrared Imaging Radiometer Suite (VIIRS) Land data products made by the Interface Data Processing System (IDPS) using the Operational (OPS) Code during the NPP era and to recommend improvements to the algorithms in the IDPS OPS code.

The Land PEATE uses a version of the MODIS Adaptive Processing System (MODAPS), NPPDAPS, that has been modified to produce products from the IDPS OPS code and software provided by the VIIRS Science Team, and uses the MODIS Land Data Operational Product Evaluation (LDOPE) team for evaluation of the data records generated by the NPPDAPS. Land PEATE evaluates the algorithms by comparing data products generated using different versions of the algorithm and also by comparing to heritage products generated from different instrument such as MODIS using various quality assessment tools developed at LDOPE. This paper describes the Land PEATE system and some of the approaches used by the Land PEATE for evaluating the VIIRS Land algorithms during the pre-launch period of the NPP mission and the proposed plan for long term monitoring of the quality of the VIIRS Land products postlaunch.
\end{abstract}

Index Terms - Remote Sensing, NPOESS, VIIRS, Environmental Data Records, Climate Data Records

\section{INTRODUCTION}

NPP is a joint mission among the NPOESS Integrated Program Office (IPO), the National Oceanic and Atmosphere Administration (NOAA), and the National Aeronautics and Space Administration (NASA). The purpose of the mission is to provide NASA with a bridge for continuing the global change observations of the Earth Observing System (EOS) that began with the EOS Terra,
Aqua and Aura Satellite Missions. It will also provide the NPOESS operational community, including NOAA and Department of Defense (DOD), with a pre-operational risk reduction demonstration and validation for selected NPOESS instruments, science algorithms, and ground processing.

The NPP satellite is scheduled to be launched into a circular sun-synchronous polar orbit similar to that of the current EOS satellites in fall 2011. The VIIRS on-board the NPP will acquire measurements from land, ocean and atmosphere in 16 moderate resolution bands $(750 \mathrm{~m}), 5$ image resolution bands $(375 \mathrm{~m})$ and one day/night band $(750 \mathrm{~m})$ providing daily global coverage. The raw sensor data acquired by these instruments are ingested and processed using the operational software at the IDPS to produce various types of science data records. These data records are distributed to the meteorological centers and archived at the Archive and Distribution Segment (ADS) of the NPP system. The Science Data Segment (SDS) [4, 5] of the NPP system provides the NPP mission with the capability to assess the quality of the Raw Data Records (RDR) it receives from the IDPS, and the quality of the Sensor Data Records (SDR) and the Environmental Data Records (EDR) it receives from the ADS. The SDS is composed of SDS Data Distribution and Depository Element (SD3E) [2], the Integration and Test System Element (I\&TSE), the Project Science Office Element (PSOE), the NPP Instrument Calibration Support Element (NICSE), and five separate PEATEs dedicated to assessing the quality of products from the Land, Ocean, Atmosphere, Ozone, and atmospheric Sounder disciplines.

The Land PEATE will fulfill the NPP mission's goal of continuing the evaluation of science products that are main sources of the global change observations that began with the MODIS Land data from the Terra and Aqua satellites. The Land PEATE will assess the VIIRS EDRs made by IDPS using the Operational Code and recommend improvements needed to the algorithms in the Operational Code to make the EDRs equivalent to the Climate Data Records (CDRs).

\section{LAND PEATE SYSTEM ARCHITECTURE}

The Land PEATE processing facility NPPDAPS, is a modified version of MODAPS and shares the MODIS 
LDOPE team for evaluation of the VIIRS Land data products generated by the NPPDAPS. Figure 2.1 shows a high level interface of Land PEATE to external NPP mission segments and other elements within the SDS. Land PEATE will receive the IDPS operational algorithm codes from a repository in CasaNOSA, and acquire data records from the SD3E [2] and also from the ADS on ad_hoc basis. SD3E provides temporary local storage for a minimum of 32 days of xDR data and 7 days of Intermediate Products (IPs). Land PEATE will coordinate with the NICSE to run tests to verify the calibration enhancements. Land Science Team members can receive the operational land algorithms and the test results from the Land PEATE for evaluation and also can submit improved algorithms for testing and evaluation at Land PEATE. Algorithm and calibration change recommendations are reviewed by the PSOE prior to submitting them to the NPOESS Algorithm Change Control Board (CCB). Land PEATE will also have the capability to generate the required subsets for evaluation of data from the Cloud's and the Earth's Radiant Energy System (CERES) instrument which will monitor Earth's radiation budget.

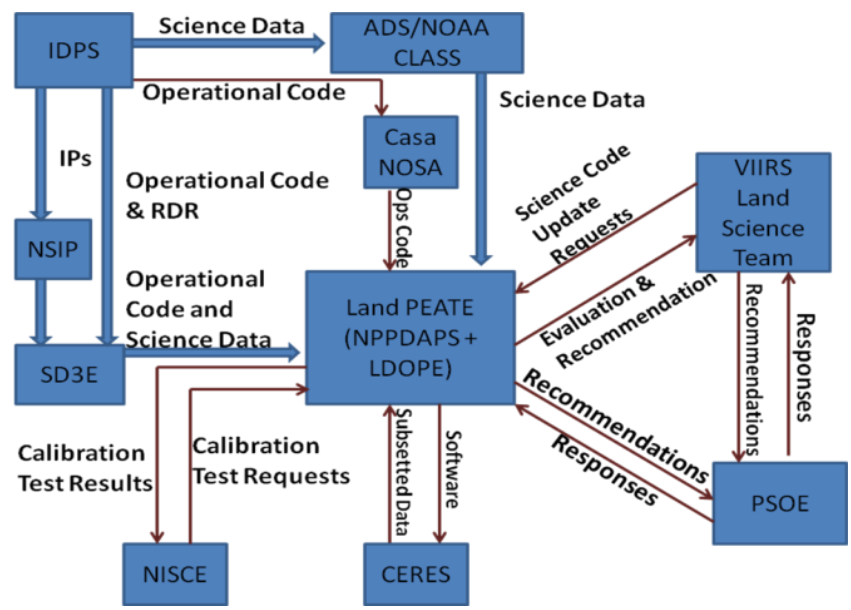

Figure 2.1: Interface of Land PEATE with SDS Elements and External Segments

The MODAPS data processing software developed to produce global MODIS science product is being used to process the VIIRS data for the Land PEATE. IDPS OPS software modules are repackaged into Product Generation Executives (PGEs) to produce the VIIRS science data products using the MODAPS data processing software. The IDPS OPS software modules are designed to run in a large shared memory system, reading and writing data entirely in memory and passing pointers to memory structures between software modules. One step in the conversion of OPS software to PGEs is to add wrapper software to each module that writes intermediate products to disk rather retaining them in memory so that the OPS code can run on small Linux servers with limited memory. In order to meaningfully compare output to the heritage MODIS products, the Land
PEATE will generate products in HDF4 formats. Land PEATE will also perform temporal aggregation of the IDPSsize swath granules containing 48 scans with temporal coverage of about 86 seconds into swath granules containing about 144 scans with temporal coverage of approximately 5 minutes.

PGEs integrated to run on NPPDAPs fall into one of the three main algorithm types identifying the source of algorithm: 1) Science Code (SCI) developed by the Northrop Grumman Aerospace Systems (NGAS) science algorithm team, 2) IDPS Operational code (OPS) developed at the IDPS by porting the SCI code to run on IDPS and 3) Science Team code (ST) developed by the VIIRS Land science teams. Land PEATE may also update any of these PGEs in response to recommendation from LDOPE or science teams. Algorithm versions updated by Land PEATE are called Land PEATE Adjusted versions (LPA). The different versions of the algorithms are identified and tracked by the Land PEATE Configuration Management (CM). The versioning attribute metadata is used to trace an algorithm back to its original source, identify changes made to enable it to run under NPPDAPS, and to evaluate its products at any stage.

\section{TESTING AND EVALUATION OF LAND ALGORITHMS}

This section describes the Land PEATE's approach to testing and evaluation of the VIIRS algorithms and presents results from testing of the algorithm versions recently released by the IDPS. Note that the examples presented here are for illustration purpose only. The final version of the VIIRS algorithm will not be available until after the post launch calibration and validation period.

During the pre-launch period, Land PEATE is using the MODIS proxy data as input in the integration testing of the individual PGEs and the science tests run to evaluate the OPS algorithms. The MODIS proxy data is generated by a proxy generator which samples the MODIS Level 1B data into VIIRS geometry and maps the MODIS bands to the nearest VIIRS bands. Land PEATE now has 51 days of MODIS proxy data enough to test all of the IDPS OPS PGEs. Land PEATE also produces a VIIRS equivalent of the MODIS Level 3 daily and multi-day gridded products, Diagnostic Data Records (DDRs) to facilitate comparison and trending between the two instruments.

Land PEATE completed integration of the build 23 (B23) (V1.5.0.23) version of the IDPS OPS algorithms in 2009 and ran a 51-day chain test generating full suite of the VIIRS Land products globally. The products included land surface reflectance, land surface temperature, land albedo, vegetation index, active fires, snow/ice cover, ice surface temperature, ice age, ice quality, cloud mask, and aerosol particles. Performance of the algorithms was evaluated by comparing the test results to the Collection 5 (C5) MODIS 
products. Build 48 (B48) (V1.5.0.48) version of the OPS algorithms was released by the IDPS in late 2009 and is currently being integrated and evaluated at the Land PEATE. Improvements in the B48 algorithms are being evaluated by comparing to the xDRs generated using the B25 algorithms and also to the C5 MODIS products.

The LDOPE team adopts the Quality Assessment (QA) approach used for operational QA of the MODIS Land products $[1,3]$ to evaluate the quality of the VIIRS products generated at Land PEATE. Since it is not feasible to inspect every granule produced from a chain test, LDOPE samples data granules by examination of global browses generated from the coarse resolution products. Land PEATE has developed and integrated a shared code to every L2 and L3 PGE to generate coarse spatial resolution product at $5 \mathrm{~km}$ resolution using appropriate aggregation scheme. Global browses of the products are generated by projecting the coarse data into a global coordinate system. These global browses are posted on the Land PEATE QA web site [6] to provide rapid synoptic product assessment via the internet. These global browse images are generated in JPEG or GIF format with fixed contrast stretching and color look-up tables to enable consistent comparison of products from different instruments (MODIS vs. VIIRS) and also from different versions (B48 vs. B23) of the algorithms. This web interface has been developed to support interactive selection of browse products and zooming and panning at $5 \mathrm{~km}$ resolution.

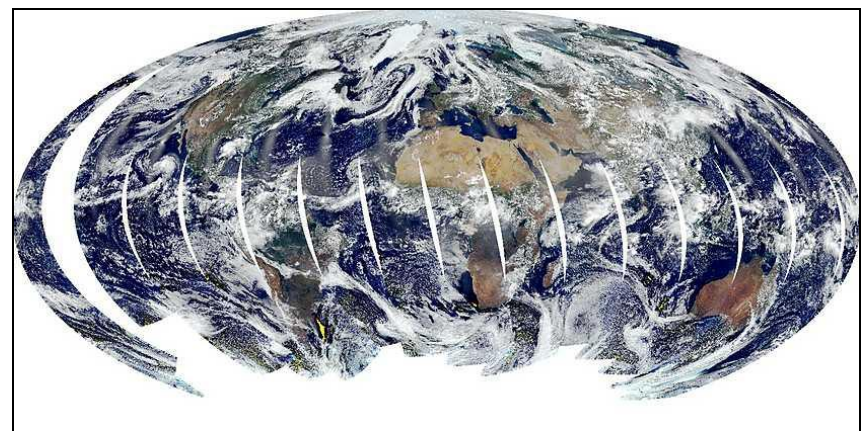

Figure 3.1: Global browse image of LSR IP from day 2003193 is an RGB composite of the bands M5 (0.672), M4 (0.555) and M3 (0.488).

Figure 3.1 illustrates global browse of the daily Land Surface Reflectance (LSR) IP produced by the B23 version of the IDPS OPS algorithm relaxed to retrieve reflectance over cloud and aerosol. The instrument sensing geometry and cloud cover are evident in the global browse image. In addition the global view of the product provides the production status and allows detection of gross product problems.

Where inspection of global browse indicates data problem or low product quality the relevant product granules are subject to more detailed assessment using the QA tools developed in-house at LDOPE. LDOPE's MODIS Land QA tools are very generic and are easily adopted for use in the quality assessment of the VIIRS Land products. These QA tools are used to compare products from different version of the OPS algorithm and also to compare VIIRS land product to the heritage MODIS product.

Figure 3.2 shows RGB composite image of the LSR IP from a 5-minute granule (day 2003193, starting time 0425) generated using the B25 and B48 LPA version of the OPS algorithm and from the MODIS C5 algorithm. Product from the B48 version shows obvious improvement compared to the B25 and also matches well with the MODIS C5 product. This improvement in B48 has been attributed to the improvement in the Aerosol Optical Thickness IP, an input used in the atmospheric correction of the surface reflectance. One way to compare the products from two different version of the algorithm or instrument is by computing the histogram of difference in the retrieved science parameters. Figure 3.3 is an example of comparing the LSR IP from the B48 VIIRS algorithm to the product from the MODIS C5 algorithm using histogram of difference in LSR.
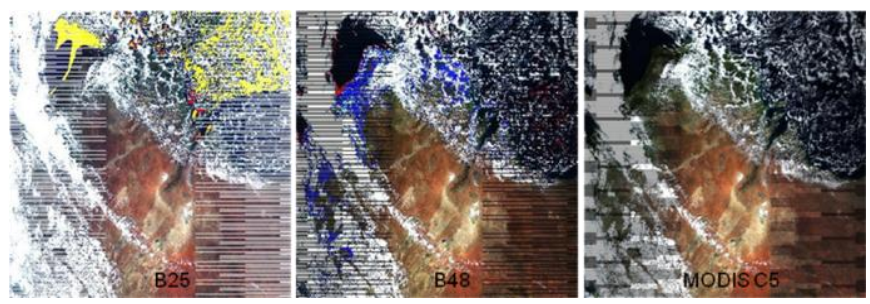

Figure 3.2: RGB composite image of bands M5, M4 and M3 in the LSR IP from B25 and B48 version of the LPA algorithm and RGB image of LSR from MODIS C5.

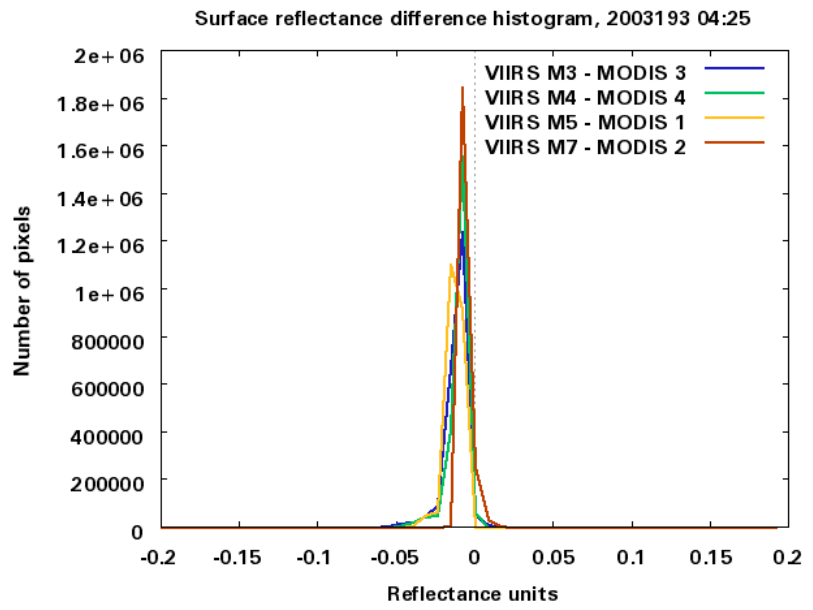

Figure 3.3: Histogram of difference used to compare LSR IP from the OPS B48 version to the MODIS C5

Figure 3.4 is an example of comparing the VIIRS Land Surface Temperature (LST) from B48 to the LST from the MODIS C5 algorithm by computing the histogram of difference in LST. The average difference in LST is less than $1^{\circ} \mathrm{K}$. Example also shows improvement in retrieval of 
LST for in-land water area in the B48 version of the IDPS OPS algorithm.

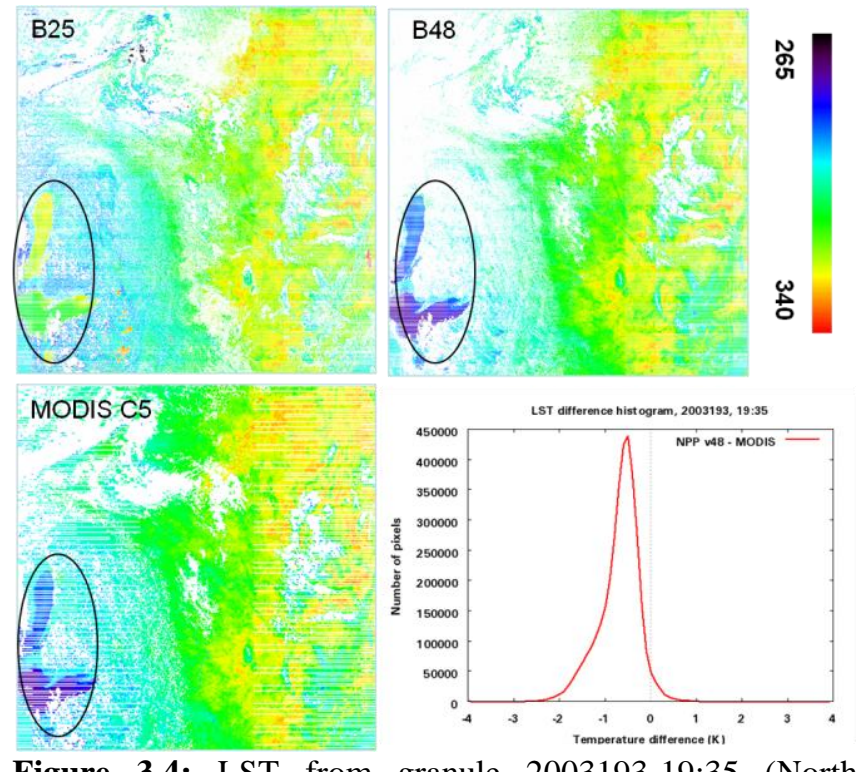

Figure 3.4: LST from granule 2003193-19:35 (North America). Circled area highlights the Great Lakes.

Product quality issues found by the LDOPE during their evaluation process are posted as known issues on the QA web site [6]. These issues are tacked and are updated as new versions of the algorithms are tested and verified by the Land PEATE.

Post-launch LDOPE will monitor the long term quality of the VIIRS Land data products by monitoring the time series statistics of the science data at a number of fixed locations of size 10deg x 10deg (golden tiles) distributed across the globe. Figure 3.5 shows the golden tiles used in the time series analysis of MODIS land products. Figure 3.6 shows time series of C5 MODIS day and night LST for savanna biome from South Africa. These product time series analyses capture algorithm sensitivity to surface (e.g. vegetation phenology), atmospheric (e.g., aerosol loading) and remote sensing (e.g., sun-surface-sensor geometry) conditions that change temporally, and they also allow changes in the instrument characteristics and calibration to be examined.

\section{SUMMARY}

The Land PEATE is an important element of the NPP Missions fulfilling the goal of continuing the evaluation of the products that are main source of the global change observation that began with the MODIS Land data from the Terra and Aqua satellites. Land PEATE so far has successfully integrated and tested three incremental build versions of the IDPS OPS algorithms and is currently working to integrate the recently released build 48. Land PEATE is expected to be fully functional meeting all the requirements and play an important role in the development of the VIIRS algorithm to generate the EDRs equivalent to the Climate Data Records (CDRs).

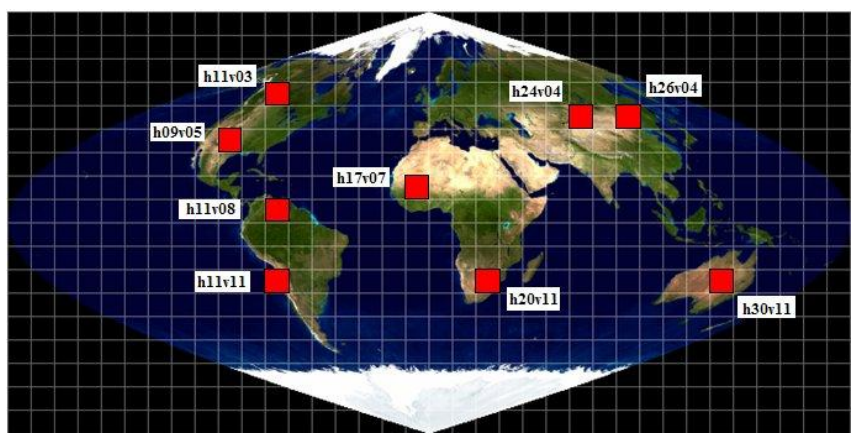

Figure 3.5: Golden tiles used in the time series analysis of MODIS Land products

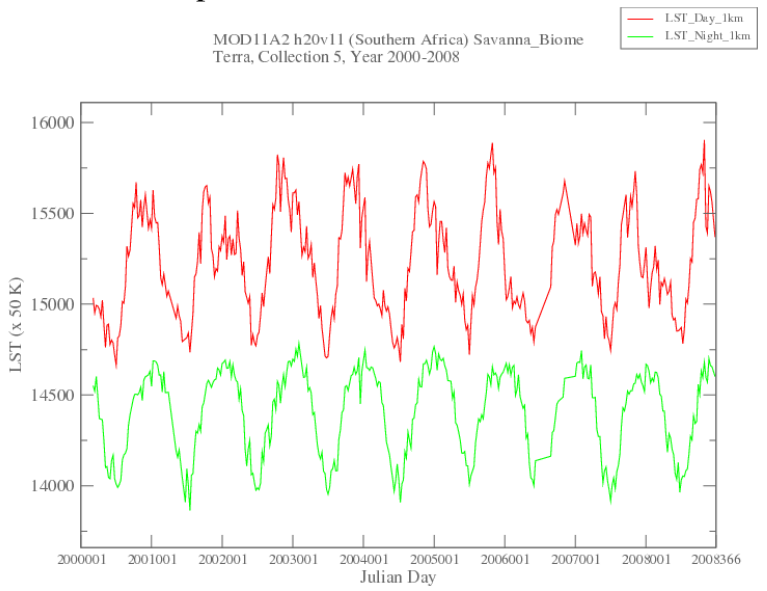

Figure 3.6: Time series of C5 MODIS LST for Savanna biome

\section{REFERENCES}

[1] David. P Roy, Jordan S. Borak, Sadashiva Devadiga, Robert E. Wolfe, Min Zheng, and Jacques Descloitres, "The MODIS Land product quality assessment approach", Remote Sensing of Environment, 83 (2002), 62-76

[2]Ho E., Schweiss R., The NPOESS Preparatory Project Science Data Segment Data Depository and Distribution Element System Architecture, IGARSS 2008, IV 613-616.

[3] Jeff Pedelty, S. Devadiga, Eric Vermote, Ed Masuoka et. al. "Generating Long-term Land Data Record from the AVHRR and MODIS Instruments, IGARSS 2007.

[4]Schweiss R., Ho E., Ullman R., Samadi S., The NPOESS Preparatory Project Science Data Segment: Brief Overview, IEEE geosciences and Remote Sensing Society, 2006

[5]Schweiss R, Hunter M., Samadi S., The NPOESS Preparatory Project Science Data Segment: The Final as Built Description, IGARSS 2008

[6] NPP Land PEATE QA Web page, http://landweb.nascom.nasa.gov/NPP 